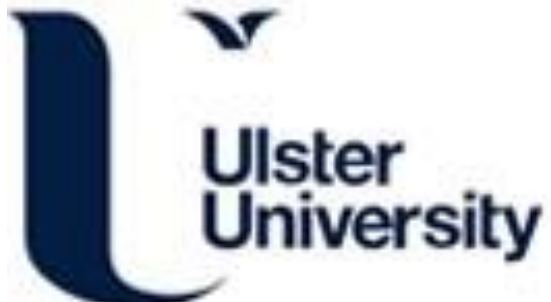

\section{Low-Loss and Tunable Localized Mid-Infrared Plasmons in Nanocrystals of Highly Degenerate InN}

Askari, S., Mariotti, D., Stehr, J. E., Benedikt, J., Keraudy, J., \& Helmersson, U. (2018). Low-Loss and Tunable Localized Mid-Infrared Plasmons in Nanocrystals of Highly Degenerate InN. Nano Letters, 18(9), 5681-5687. https://doi.org/10.1021/acs.nanolett.8b02260

Link to publication record in Ulster University Research Portal

\section{Published in: \\ Nano Letters}

Publication Status:

Published (in print/issue): 12/09/2018

DOI:

10.1021/acs.nanolett.8b02260

\section{Document Version}

Author Accepted version

\section{General rights}

Copyright for the publications made accessible via Ulster University's Research Portal is retained by the author(s) and / or other copyright owners and it is a condition of accessing these publications that users recognise and abide by the legal requirements associated with these rights.

\section{Take down policy}

The Research Portal is Ulster University's institutional repository that provides access to Ulster's research outputs. Every effort has been made to ensure that content in the Research Portal does not infringe any person's rights, or applicable UK laws. If you discover content in the Research Portal that you believe breaches copyright or violates any law, please contact pure-support@ulster.ac.uk. 


\title{
Low-loss and tunable localized mid-infrared plasmons in nanocrystals of highly-degenerate InN
}

\author{
Sadegh Askari ${ }^{1,4 *}$, Davide Mariotti ${ }^{2}$, Jan Eric Stehr ${ }^{3}$, Jan Benedikt ${ }^{4}$, Julien \\ Keraudy $^{1}$, Ulf Helmersson ${ }^{1}$ \\ ${ }^{1}$ Plasma \& Coatings Physics Division, IFM-Material Physics, Linköping University, SE-581 83 \\ Linköping, Sweden \\ ${ }^{2}$ Engineering Research Institute (ERI), Ulster University, BT37 0QB, Northern Ireland, UK \\ ${ }^{3}$ Department of Physics, Chemistry and Biology, Linköping University, $581 \quad 83$ \\ Linköping, Sweden \\ ${ }^{4}$ Institute for Experimental and Applied Physics, Christian-Albrechts-Universität zu Kiel, \\ Leibnizstraße 17, 24118 Kiel, Germany
}

\begin{abstract}
Plasmonic response of free charges confined in nanostructures of plasmonic materials is a powerful means for manipulating the light-material interaction at the nanoscale, and hence has influence on various relevant technologies. In particular, plasmonic materials responsive in the mid-infrared range are technologically important as the mid-infrared is home to the vibrational resonance of molecules and also thermal radiation of hot objects. However, the development of the field is practically challenged with the lack of low-loss materials supporting high quality plasmons in this range of the spectrum. Here we demonstrate that degenerately doped InN nanocrystals (NCs) support tunable and low-loss plasmon resonance spanning the entire mid-wave infrared range. Modulating free-carrier concentration is achieved by engineering nitrogen-vacancy defects $\left(\operatorname{InN}_{1-\mathrm{x}}, 0.017<\mathrm{x}<0.085\right)$ in highly degenerate NCs using a non-equilibrium gas-phase growth process. Despite the significant reduction in the carrier mobility relative to intrinsic $\mathrm{InN}$, the mobility in
\end{abstract}


degenerate InN NCs $\left(>60 \mathrm{~cm}^{2} / \mathrm{Vs}\right)$ remains considerably higher than the carrier mobility reported for other materials NCs such as doped metal oxides, chalcogenides and noble metals. These findings demonstrate feasibility of controlled tuning of infrared plasmon resonances in a low-loss material of III-V compounds and open a gateway to further studies of these materials nanostructures for infrared plasmonic applications.

Plasmonic materials active within the mid-infrared range enable enhancing light-material interaction in a technologically important spectral range that is home to the vibrational resonances of molecules and also thermal radiation of hot objects [1-3]. In mid-infrared responsive nanostructures, the oscillation of free carriers is confined to the scales around two orders of magnitude smaller than the light wavelength, resulting in strong and highly localized electromagnetic fields. The energy concentrated at nanoscale can be transferred into electricity (generation of "hot electrons"), coupled with the molecular vibrations, etc [1-5]. Localized surface plasmon resonances (LSPRs) active in the mid-infrared range are under study for applications such as targeted chemical sensing and spectroscopy, sensing low-dimensional bioobjects (e.g. biomolecules smaller than $10 \mathrm{~nm}$ ), thermal imaging and heat scavenging, to name a few [1-3]. Materials studied for the mid-infrared range still struggle with limitations such as restricted spectral range (graphene) [6], low mobility (doped metal oxides) [7-8] or low concentration of charge carriers (InAs, GaN and Si) [9]. There is an urgent need of finding lowloss materials with tunable plasmonic responses and a high free carrier concentration to fully unlock the potential of plasmonics in the mid-infrared range [2, 10, 11]. Compounds of group III-V semiconductors (e.g. InAs, GaN, GaP) are studied for plasmonic applications due to their small effective electron mass which results in a high electron mobility. However, high doping (carrier concentration $>10^{20} \mathrm{~cm}^{-3}$ ) of these semiconductors is not easily achievable and the plasmonic resonances reported for these materials are limited to very low energies [7]. An attractive and less-studied group III-V compound is InN that has a very low effective electron mass (lowest among the nitride semiconductors) and strong propensity for n-type conductivity which makes excellent combination for an ideal plasmonic material. A wide variation of the carrier concentration, from $10^{17} \mathrm{~cm}^{-3}$ to more than $10^{21} \mathrm{~cm}^{-3}$, has been reported for thin films of InN 
[13]. The high concentration of free electrons in $\mathrm{InN}$ is explained by the special characteristic of its bandgap in which the charge neutrality level is located deep in the conduction band; i.e. about $1.83 \mathrm{eV}$ above the valence band maximum while the conduction band minimum is $0.67 \mathrm{eV}$ above the valence band maximum [14]. Defect and impurities in InN are therefore generally of the donor type. In particular, nitrogen vacancies have the lowest formation energy among the native point defects in $\mathrm{InN}$ and thus are considered as a possible cause of n-type conductivity especially when the growth of $\mathrm{InN}$ is performed using a non-equilibrium growth process [15].

We have investigated the plasmonic response of the degenerately doped $\mathrm{InN}$ nanocrystals with varying degree of nitrogen deficiency. Growth of $\mathrm{InN}$ nanomaterials by conventional chemical methods is very challenging due to the lack of suitable precursors and the covalent nature of the bonds which complicates control over the nucleation and growth processes [16]; i.e. previous reports on the synthesis of InN NCs have proven the incompatibility of solution-based methods for controlled growth of these materials [17-20]. In fact, $\mathrm{InN}$ is one of the least understood of the III-V semiconductors, mostly due to the difficulties associated with its preparation both in the form of thin films and as nanomaterials [16-22]. Synthesis methods based on non-thermal plasmas are compatible with growth of semiconductors with more covalent bounds [23]. Here, we employ a novel plasma-based technique that is compatible with growth of ionically bound semiconductors and allows versatile control of the chemical composition and size of the NCs. 

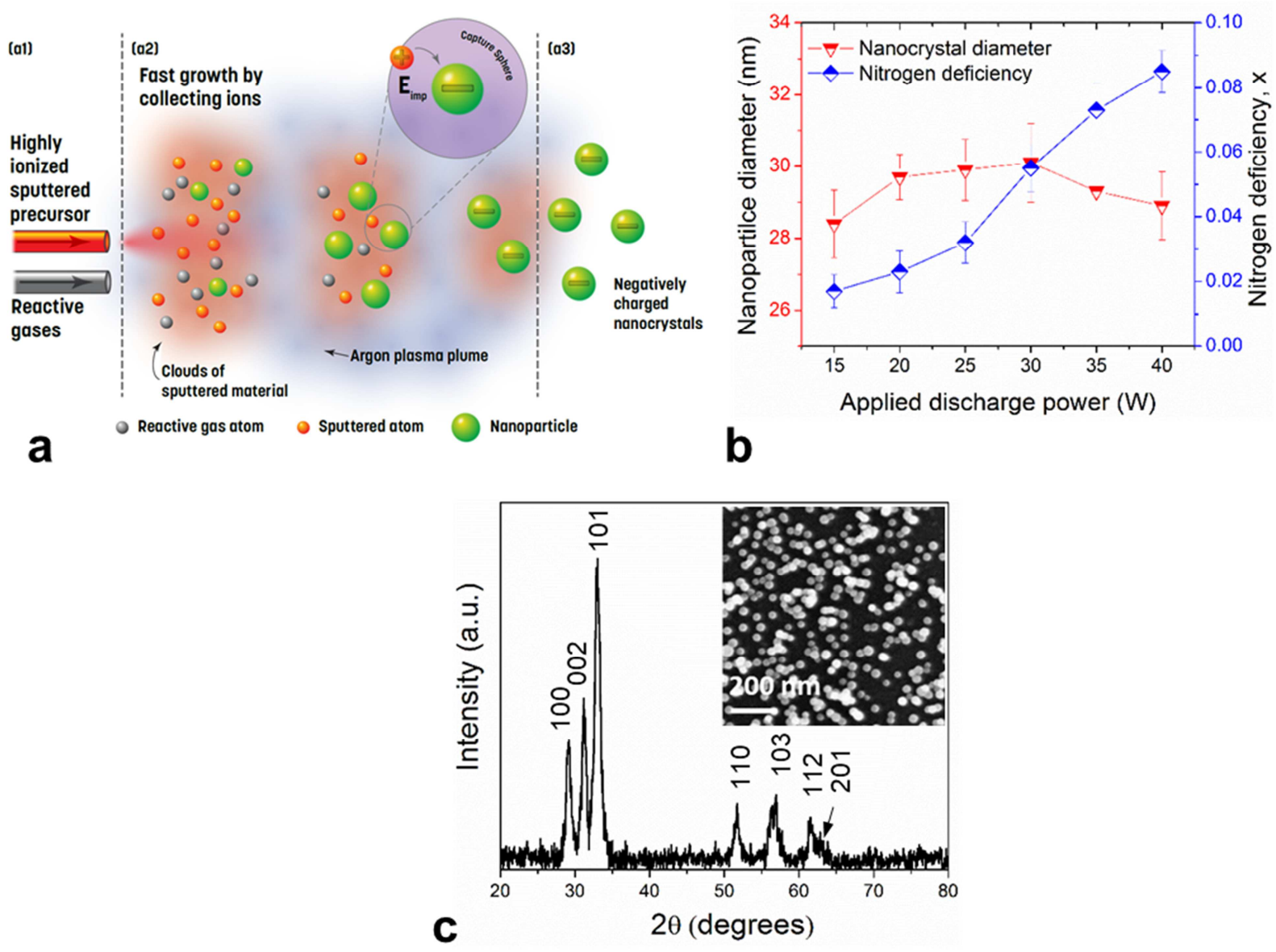

Figure 1: a, Pulsed plasma process for controlled growth of nanocrystals (NCs). The generated plasma is highly ionized and lead to high growth rates by collecting ions into the negatively charged NCs. $\mathrm{E}_{\text {imp }}$ denotes the kinetic energy of ions impinging on the NC. $\mathbf{b}$, Nanocrystal diameter and corresponding nitrogen deficiency for NCs produced by varying the average plasma power; error bars for the nitrogen deficiency represents the standard error of the mean value and for the NC diamter represents the standard deviation of the distribution for the corresponding mean value. c, Typical XRD and SEM image (inset) of wurtzite InN NCs.

Figure 1a shows a schematic diagram of the process (see also section S1 in the SI document). Argon gas flows through the indium hollow cathode and the plasma is maintained by applying a pulsed electric field to the cathode which leads to sputtering of the indium cathode. Nitrogen gas added to the plasma is dissociated mainly through interaction with the highly energetic electrons in the plasma. The pulsed nature of this plasma provides a saturated vapor of highly ionized sputtered material which is favorable for the rapid growth of NCs in a non-equilibrium process 
[37-38]. Further detail of the experiment set-up and the process is presented in the SI. The growing NCs are negatively charged in the plasma due to the higher mobility of electrons (versus ions) and it results in the fast accumulation of (positively charged) ions onto the NCs. The control over nitrogen deficiency in the NCs is achieved by changing the average applied power to the cathode in the range from $15 \mathrm{~W}$ to $40 \mathrm{~W}$, which enhances indium sputtering rate and as a result the relative concentration of In to N in the NCs. The pulse frequency and length was fixed at $500 \mathrm{~Hz}$ and $80 \mu \mathrm{s}$, respectively. Figure $1 \mathrm{~b}$ shows no significant changes in the size distribution of the NCs while varying the applied power. Energy dispersive X-ray spectroscopy (EDX) analysis was used to determine the indium/nitrogen content in the NCs collected directly on substrates. For each synthesis condition, measurements were taken on multiple locations and the values averaged (figure $1 \mathrm{~b}$ ); the error bars in figure $1 \mathrm{~b}$ correspond to the standard deviation. The results show that the nitrogen is deficient in all samples $\left(\mathrm{InN}_{1-\mathrm{x}}\right)$ and the deficiency increased from $\mathrm{x}=0.017$ to $\mathrm{x}=0.085$ with increasing the average pulse power from $15 \mathrm{~W}$ to $40 \mathrm{~W}$. The measured oxygen and carbon impurities in the samples are on a constant level ( 5-6\%) independent on process conditions. We believe that these impurities mainly originates from exposure to the open atmosphere and the initial contamination of the substrate. X-ray diffraction (XRD) analysis shows that all the samples have hexagonal wurtzite structure. Figure 1c shows the XRD pattern collected for a sample prepared at applied power of $20 \mathrm{~W}$. All diffraction peaks can be indexed to the different planes of wurtzite InN and the XRD profiles did not change with nitrogen content for the range studied here. We should note that the utilized plasma process allows rapid growth of NCs without chemical ligands or impurities and excellent control of the chemical composition. It is likely the non-equilibrium interaction of highly energetic ions with the bare surfaces of the growing NCs that allows for the formation of native defects buried inside the NCs. 


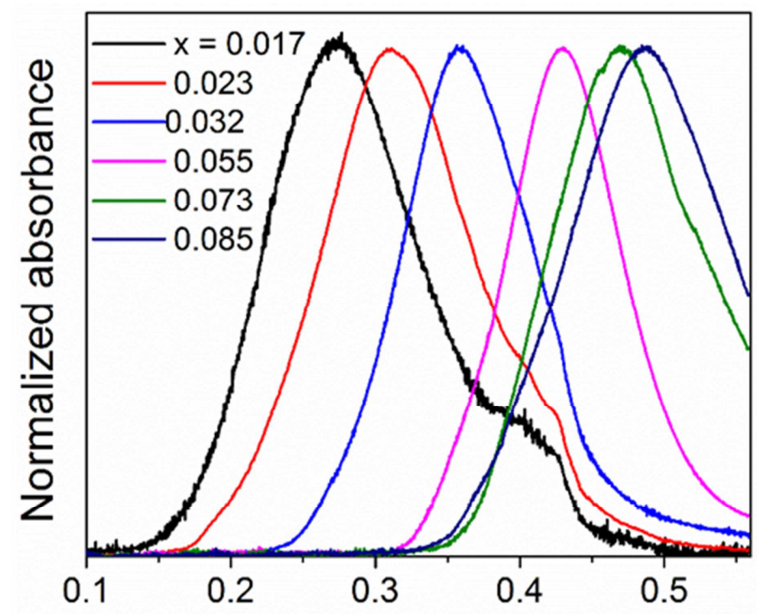

a Energy (eV)

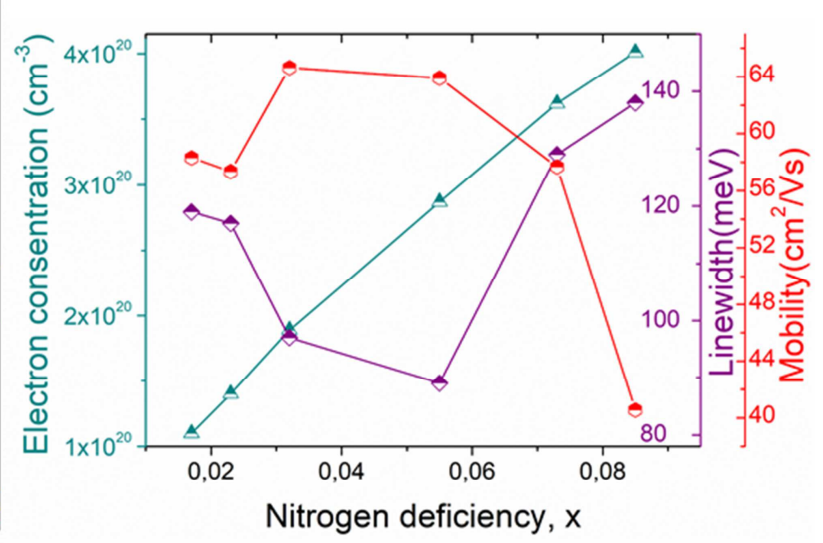

b

Figure 2: a, The FTIR spectra of the InN nanocrystals for a range of nitrogen deficiencies, $\operatorname{InN}_{1-x}$, $0.017<x<0.085$. The absorption peak is shifted to higher energies in nanocrystals with higher nitrogen deficiency. b, Free-electron concentration, damping factor and optically calculated free electron mobility as a function of nitrogen deficiency in InN nanocrystals.

Figure 2a shows the infrared absorbance spectra of the NCs with varied nitrogen deficiencies. It can be seen that the resonance peak position is shifted toward higher energies in samples with higher nitrogen deficiencies. The peak position is shifted from $0.27 \mathrm{eV}$ to $0.49 \mathrm{eV}$ with increasing nitrogen deficiency in the range $0.017<\mathrm{x}<0.085$ in $\mathrm{InN}_{1-\mathrm{x}} \mathrm{NCs}$ (figure 2a). The well-defined absorption peaks and corresponding shifts across the mid-infrared region of the spectrum can be explained by the high concentration of free electrons that is increasing in NCs with increasing nitrogen deficiency (figure $2 b$ ). The electron concentration calculated from the plasmon resonance peaks in figure $2 \mathrm{a}$ (see SI for details) ranges from $1.18 \times 10^{20} \mathrm{~cm}^{-3}$ to $3.2 \times 10^{20}$ $\mathrm{cm}^{-3}$ (figure 2b). Infrared absorption has previously been observed in $\mathrm{InN}$ thin films [24-25] and it has been attributed to the plasmon absorption by free electrons. The opto-electronic properties of InN NCs have rarely been studied. Palomaki et al. [26] has recently reported plasmon absorption for InN NCs prepared by a colloidal synthesis technique with an LSPR peak around $3000 \mathrm{~nm}(0.41 \mathrm{eV})$. They reported limited changes in the free electron concentration from $2.51 \times 10^{20} \mathrm{~cm}^{-3}$ to $2.89 \times 10^{20} \mathrm{~cm}^{-3}$ induced by chemical oxidation, which clearly highlight the stabilization of the electron concentration due to the proximity of the Fermi level to the charge neutrality level [14]. Furthermore, in this case the electron concentration was not controlled by 
the chemical composition of the actual NCs rather by implementing a junction at the interface between core InN NC and the oxidized shell.

\begin{tabular}{|c|c|c|c|c|c|}
\hline \multicolumn{2}{|c|}{$\begin{array}{c}\text { Nanocrystal } \\
\text { material }\end{array}$} & \begin{tabular}{|c|} 
Responsive \\
range
\end{tabular} & \begin{tabular}{|c|} 
Damping \\
factor $(\mathrm{meV})$
\end{tabular} & \begin{tabular}{|c|} 
Effective electron \\
mass (electron mass)
\end{tabular} & $\begin{array}{c}\text { Electron Mobility } \\
\left(\mathrm{cm}^{2} / \mathrm{Vs}\right)\end{array}$ \\
\hline \multicolumn{2}{|c|}{$\ln \mathrm{N}^{\text {[this work] }}$} & Infrared & 89 & $0.03-0.25$ & 65 \\
\hline \multicolumn{2}{|c|}{$\mathrm{Au}^{[28]}$} & visible & 120 & 1.1 & \\
\hline \multicolumn{2}{|c|}{$\mathrm{Cu}_{2-x} \mathrm{~S}^{[27]}$} & Near-infrared & 210 & 0.8 & 6.9 \\
\hline \multirow{4}{*}{ 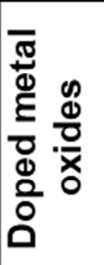 } & $\mathrm{Al}: \mathrm{ZnO}[29]$ & Infrared & $\sim 270$ & 0.38 & $\sim 11.0$ \\
\hline & $\mathrm{Sn}: \ln _{2} \mathrm{O}_{3}[30]$ & Infrared & 113 & 0.38 & 19.9 \\
\hline & $\mathrm{Ce}: \ln _{2} \mathrm{O}_{3}{ }^{[31]}$ & Infrared & 77 & $\sim 0.38$ & 33.0 \\
\hline & In\&F:CdO[32] & Near-infrared & 59 & & \\
\hline
\end{tabular}

Table 1: Comparison of plasmonic nanocrystals responsive in different range of spectrum.

The linewidth of the absorbance profiles in figure $2 \mathrm{a}$ is proportional to the damping of free electrons due to scattering and thus can be used for determining electron mobility (see sections $\mathrm{S} 3$ in the SI). The narrow linewidth of the absorbance profiles with full width half maximum (FWHM) down to $89 \mathrm{meV}$ is among the narrowest linewidths reported for plasmonic NCs (see table 1); this strongly supports the suitability and attractiveness of $\mathrm{InN}$ as a plasmonic material. Electron mobility calculated from the linewidth of the absorbance profiles is presented in figure 2b. Electron mobility up to a value of $64.8 \mathrm{~cm}^{2} / \mathrm{Vs}$ is obtained which is higher than the previously reported carrier mobility for any plasmonic NC including doped metal oxides, chalcogenides and noble metals. Table 1 compares InN NCs damping factor and mobility with several other well-studied plasmonic NCs. Recent studies shows that defect engineering of doped metal oxide can lead to an electron mobility as high as $33 \mathrm{~cm}^{2} / \mathrm{Vs}$ (in Ce doped $\operatorname{In}_{2} \mathrm{O}_{3} \mathrm{NCs}$ [31]). Electron mobility for InN NCs remains considerably higher than other plasmonic NCs owing to the non-parabolic nature of InN conduction band with values of low effective electron mass. To understand the exceptional properties of InN NCs in this context, we will next discuss the effect of changing the nitrogen content on the energy band diagram of the NCs, including Fermi level and optical bandgap. 

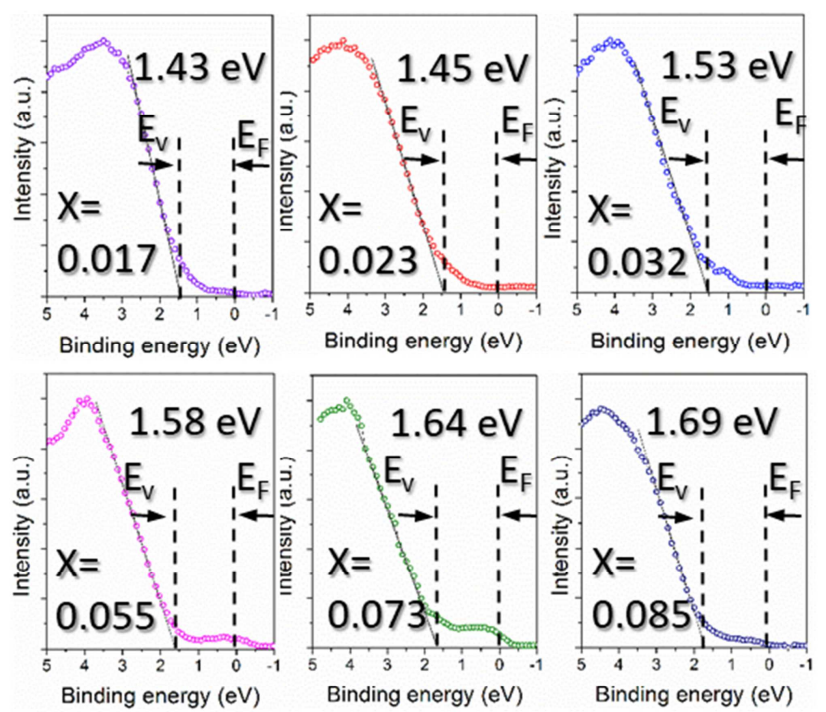

a
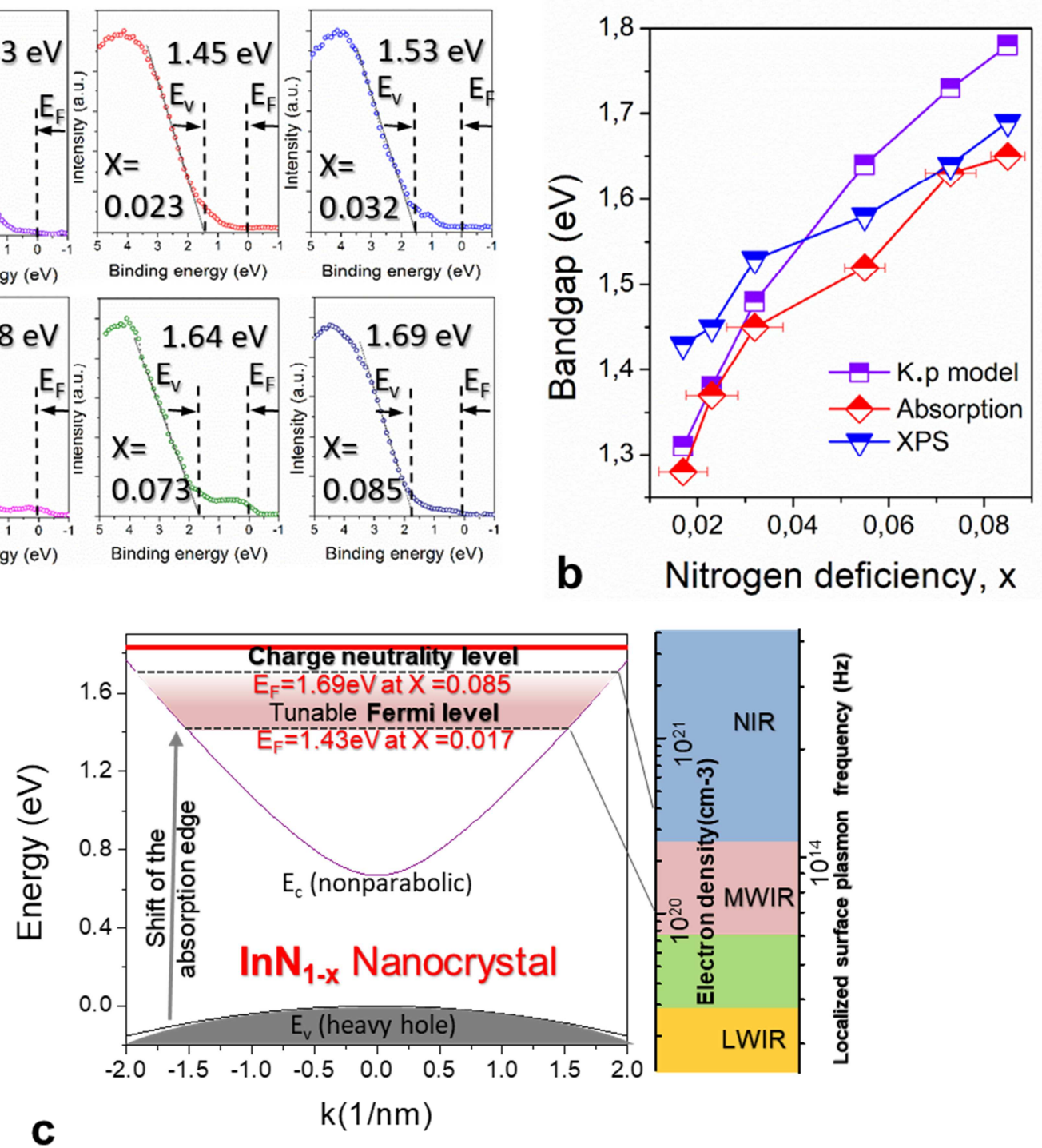

Figure 3: a, XPS valence band spectra of $\mathrm{InN}$ nanocrystals with different nitrogen deficiency, $\operatorname{InN}_{1-x}$, $0.017<x<0.085$. The difference between the valence band maximum and Fermi level is increased in nanocrystals with higher nitrogen deficiency. $\mathbf{b}$, The optical bandgap in InN nanocrystals as a function of nitrogen deficiency is obtained from the ultraviolet-visible absorption measurements, XPS measurements (fig3a) and calculated from Kane model. c, Modulating free-electron concentration and Fermi level in degenerate $\mathrm{InN}_{1-\mathrm{x}}$ nanocrystals. The right panel shows localized surface plasmon frequency versus freeelectron concentration. The technically important infrared ranges of spectrum are shown NIR (nearinfrared), MWIR (mid-wave infrared) and LWIR (long-wave infrared). 
The valence band spectra obtained from XPS measurements are shown in Figure 3a. The Fermi level relative to the valence band maximum is shifted from $1.43 \mathrm{eV}$ to $1.69 \mathrm{eV}$ with increasing nitrogen deficiency of NCs, i.e. the Fermi level is located deeply in the conduction band (the conduction band minimum is located at $0.67 \mathrm{eV}$ from the valence band edge) and it approaches charge neutrality level in NCs with higher nitrogen deficiency (figure $3 \mathrm{c}$ ). It has been previously observed that the Fermi level is stabilized where the free electron concentration reaches its maximum saturated value at very high concentration of defects [14], however the Fermi level is here well below saturation so that varying the nitrogen deficiency can effectively shift the plasmon resonance peak. The high-energy shift of the Fermi level is in agreement with the widening of the optical bandgap obtained from the ultraviolet-visible absorption measurements (figure 3b). The bandgap is determined by extrapolating the linear part of the squared absorption coefficient (see figure S5 in SI). The bandgap is increased from $1.28 \mathrm{eV}$ to $1.65 \mathrm{eV}$ in NCs with higher nitrogen deficiency (figure 2b). The values obtained for the bandgap from XPS (i.e. from figure $2 a$ ) is also included in figure $2 b$ for an easier and direct comparison with absorption measurements. XPS measurements produced slightly higher values and this can be explained with the downward band bending due to the surface charge accumulation effect which has been previously observed in InN thin films [14]. However, we note that the difference is small and well within experimental errors or due to intrinsic differences in the measurement and fitting techniques. The blue shift of the absorption edge in degenerate semiconductors could be due to the Burstein-Moss (BM) effect. The BM shift in $\mathrm{InN}$ can be calculated from the dispersion relation for the conduction band obtained from a two-band $\boldsymbol{k} \cdot \boldsymbol{p}$ perturbation model presented by Kane [33],

$$
E_{c}(k)=E_{g}+\frac{\hbar^{2} k^{2}}{2 m_{0}}+\frac{1}{2}\left(\sqrt{E_{g}{ }^{2}-4 E_{p} \cdot \frac{\hbar^{2} k^{2}}{2 m_{0}}}-E_{g}\right)
$$

where $E_{g}=0.67 \mathrm{eV}$ is the bandgap of $\mathrm{InN}, k$ is the wave vector, $m_{0}$ is the free electron mass. $E_{p}$ is an energy parameter of the Kane's model and it is taken $E_{p}=10$. InN, as a narrow bandgap semiconductor, has a strongly non-parabolic conduction band due to the $\boldsymbol{k} . \boldsymbol{p}$ interaction between valence and conduction bands. This non-parabolicity has been taken into account in the Kane model (eq. 1). The Fermi level is given by eq. 1 evaluated at the Fermi wave vector $k_{F}=$ $\left(3 \pi^{2} n_{e}\right)^{1 / 3}$. The concentration of free electrons $n_{e}$ in plasmonic NCs can be estimated from the 
correlation between the bulk plasma oscillation frequency and the plasmon resonance frequency (see section S3 in SI and figure $2 b$ ). Figure 3 b shows the calculated bandgap energy from eq. 1 for the NCs with different nitrogen deficiency. The good agreement of the predicted BM shift with the absorption measurements is further evidence that the widening of the optical bandgap is due to the high density of free electrons. Importantly, the consistency of the results for the Fermi level and the optical gap with the BM predictions certifies that the values for electron concentration calculated from the plasmon absorption peaks are accurate.

We finally discuss the possible origin of our measured electron concentrations and further corroborate the contribution from nitrogen deficiencies. In InN, the Fermi level lies far below the charge neutrality level even in degenerate $\mathrm{InN}$, so that donor-type defects such as nitrogen vacancies are energetically favorable. Particularly nitrogen vacancies can play role as major defects in materials similar to $\mathrm{InN}$ such as $\mathrm{GaN}$ and $\mathrm{AlN}$ [34-35]. In InN, nitrogen vacancies have the lowest formation energies among all native defects [15, 36]. Hydrogen impurities incorporated into the lattice during the growth process can also effectively act as electron donors in InN, however leading to much lower concentrations of free carriers $\left(<<10^{20} \mathrm{~cm}^{-3}\right)$ [36]. The higher concentration of free electrons observed with increasing nitrogen deficiency in NCs is evidence of the effective presence of electrically active nitrogen vacancies. The stable charge state of the nitrogen vacancy is $3+$ for Fermi level (relative to the valence band maximum) below $0.24 \mathrm{eV}$ and $1+$ for higher Fermi levels. The formation energy of $V_{N}{ }^{+}$in the bulk InN with the Fermi level being located at the conduction band minimum is $1.0 \mathrm{eV}$ and its increasing with increasing the Fermi level. However, this formation energy is considerably reduced on the surface of NCs during the growth so that it can be even negative $(\sim-0.13 \mathrm{eV}$ [15]) under In-rich growth conditions.

The high concentration of defects (donors and acceptors) observed in films of InN grown by different techniques have not been well explained by numerical calculations based on the formation energies with assuming thermodynamic equilibrium condition, i.e. the formation energies are too high. A possible reason is the deviation of the growth processes from equilibrium especially when the non-equilibrium plasma processes are involved. The nonequilibrium growth of thin films with a typical growth rate of $0.1 \mathrm{~nm} / \mathrm{s}$ can lead to a nitrogen surface vacancy concentration of $2.1 \times 10^{20} \mathrm{~cm}^{-3}$ extending several tens of $\mathrm{nm}$ into the bulk [15]. 
The fast non-equilibrium growth of NCs in the plasmas with the presence of highly energetic and reactive species allows the formation of nitrogen vacancy defects. A typical growth rate of NCs is much higher than the growth rate of thin films owing to the accretion of positively charged ions trapped by the negatively charged NCs inside the plasma. For instance, we have previously reported growth rate of $\sim 500 \mathrm{~nm} / \mathrm{s}$ for copper NCs [37] which is several orders of magnitude higher than the typical growth rate of films $(\sim 0.1 \mathrm{~nm} / \mathrm{s})$. The ion concentration in pulsed plasma is significantly higher than the neutrals concentration ( $>80 \%$ ions [38]) and the positive ions are accelerated to the nanoparticle over a potential that allow them to gain a kinetic energy of $\mathrm{E}_{\mathrm{imp}} \sim 2.8 \mathrm{eV}$ for singly charged ions, typically higher than the formation energy of the nitrogen vacancy defect. Under the rapid non-equilibrium growth condition of the NPs, defects formed on the surface during the growth are buried inside the NC. The concentration of vacancies can be higher at the surface of NCs which can lead to higher electron concentration on the surface similar to the surface charge accumulation widely observed in thin films of $\mathrm{InN}$.
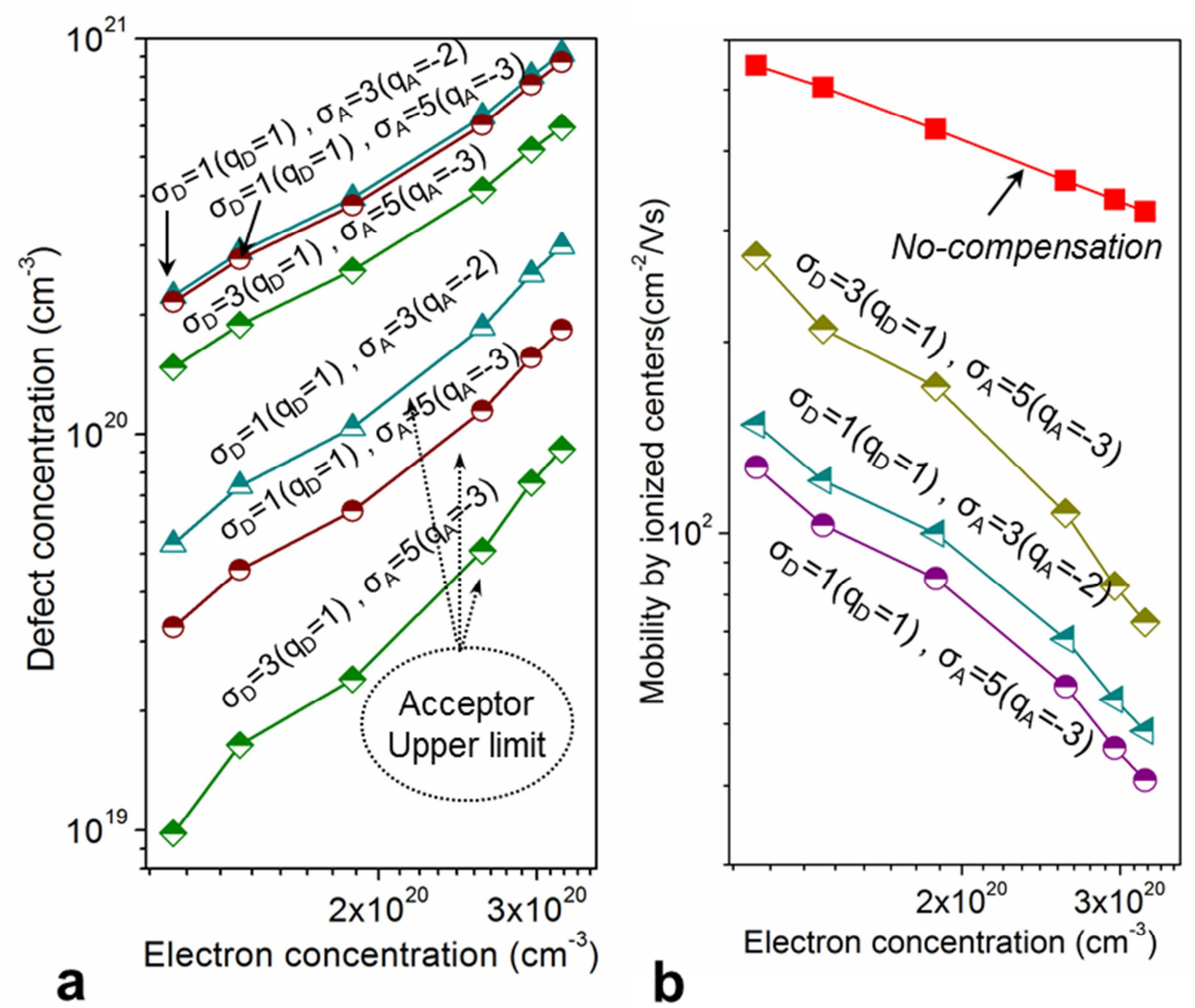
Figure 4: a, Concentration of donor and acceptor nitrogen vacancy defects in InN nanocrystals (see SI for details). b, Calculated mobility limited by ionized defect centers in $\mathrm{InN}$ presented as a function of electron concentration.

At high Fermi levels, the acceptor defects become more favorable so that intense compensation occurs in highly degenerate $\mathrm{InN}$ [39-41]. Nitrogen vacancies in highly degenerate $\mathrm{InN}$ have also a tendency towards forming clusters (or complexes) of vacancies with a reduction in the absolute defect charge [37]. Density-functional theory calculations show that the clusters of nitrogen vacancies with neutral charge state become favorable [41]. Furthermore, formation of positively charged complexes is expected with a lower charge state. The concentration of the nitrogen vacancy defects can be evaluated with considering more favorable vacancies with different charge states. In the calculations, charge states of $\mathrm{q}_{\mathrm{D}}$ (donors) and $\mathrm{q}_{\mathrm{A}}$ (acceptors) with the highest stability are considered (see section S5 in the SI document). The concentration of donors for the most favorable vacancies calculated for the NCs with different nitrogen deficiencies are shown in figure $4 \mathrm{a}$. The figure also includes the upper limit obtained for the acceptor concentration.

Carrier mobility in $\mathrm{InN}$ is reduced with increasing free electron concentration from a few thousands $\mathrm{cm}^{2} / \mathrm{Vs}$ at low electron concentrations $\left(\sim 10^{17} \mathrm{~cm}^{-3}\right)$ to a few hundred $\mathrm{cm}^{2} / \mathrm{Vs}$ and less at high electron concentrations $\left(>10^{20} \mathrm{~cm}^{-3}\right)$ mainly due to the scattering from ionized defect centers [39, 42]. The electron mobility due to the ionized defect scattering can be calculated from the known value of the defect concentrations (section S6 in the SI file). The calculated electron mobility corresponds to these defect concentrations are presented in figure $4 \mathrm{~b}$. Electron mobility where the concentration of acceptors is negligible, no-compensation, is several times higher than the values obtained from the damping factor (figure $4 \mathrm{~b}$ ). Therefore significant compensation with an acceptor concentration likely lower than the free electron concentration in NCs is expected.

This article introduces $\mathrm{InN}$ as a promising low-loss plasmonic material. Defect engineering of degenerately doped $\mathrm{InN}$ allows controlled modulation of the free carrier concentration to values higher than $10^{20} \mathrm{~cm}^{-3}$. At such a high doping levels, the optical bandgap is significantly widened, Fermi level is shifted deeply into the conduction band, and an intense plasmon absorption band appears in the infrared range of the spectrum. Carrier concentration in our NCs could be efficiently modulated by changing nitrogen vacancy defects through using a non-equilibrium 
plasma-based growth process. We found plasmon resonance absorption with narrow linewidth and tunable peaks across the mid-infrared spectral range. The very high electron mobility of these InN NCs well above carrier mobility observed in other doped semiconductors is highly attractive toward resolving the challenge of losses in plasmonic materials. This study opens a pathway for further research of doped $\mathrm{InN}$ and similar semiconductors (from group III-V) nanostructures as a new class of plasmonic material.

\section{Methods}

Nanocrystals (NCs) are synthesized using a custom-made set-up based on high-power pulsed plasma sputtering technique. The detailed description of the process and the experimental set-up is presented in the SI (section S1). For all the samples reported here, the operating pressure was 0.8 Torr and the argon and nitrogen gas flow rates were $80 \mathrm{sccm}$ and $20 \mathrm{sccm}$, respectively. Control over stocihiometry in nitrogent deficient NPs $\left(\operatorname{InN}_{1-\mathrm{x}}, 0.017<\mathrm{x}<0.085\right)$ is achived by changing the applied power in the range from $15 \mathrm{~W}$ to $40 \mathrm{~W}$ while the other operating parameters are held constant. The NCs produced in the plasma are collected downstream of the reactor directly onto the substrates for characterizations. All measurements presented here were carried out shortly after synthesis therefore preserving the as-synthesized surface characteristics.

Mean diameter of the NCs and size distributions are determined by scanning electron microscopy (SEM) analysis. Measurements are performed with a LEO 1550 Gemini equipped with an energy dispersive X-ray spectroscopy (EDX) detector (Oxford instruments). The EDX analysis are carried out by data collected from three different spots on each sample.

The X-ray diffraction (XRD) measurements are performed using a PANalytical X'pert diffractometer mounted with a hybrid monochromator/mirror, operated at $40 \mathrm{kV}$ and $40 \mathrm{~mA}$ with a $\mathrm{Cu}$ anode $(\mathrm{Cu} \mathrm{K \alpha}, \lambda=1.540597 \AA)$. For typical XRD measurements, a thick layer (to avoid substrate peaks) of NCs are deposited on metal coated silicon substrates.

UV-vis absorption measurements are performed using a Perkin-Elmer, Lambda 950 UV-VIS spectrometer; samples for UV-vis measurements are collected on glass substrates.

The Fourier transform infrared (FTIR) spectroscopy is carried out using an attenuated total reflectance (ATR) accessory. The ATR-FTIR spectrometer is a Bruker Vertex 70 FTIR 
spectrometer, and for the measurements, the collected sample powder on a metal coated silicon substrate is placed on the top of the ATR crystal.

X-ray photoelectron spectroscopy (XPS) measurements are performed using an Kratos Ultra photoelectron spectrometer equipped with a monochromated $\mathrm{Al} \mathrm{K} \alpha(1486.6 \mathrm{eV})$ x-ray source operating at $150 \mathrm{~W}$. The carbon peak $\mathrm{C} 1 \mathrm{~s}$ at $284.3 \mathrm{eV}$ was used for calibrating the spectra.

\section{Acknowledgement}

The authors would like to thank the Knut and Alice Wallenberg Foundation (grant KAW 14.0276) and the Swedish Government Strategic Research Area in Materials Science on Functional Materials at Linköping University (faculty grant SFO-Mat-LiU \#200900971) for financial support.

\section{References:}

1] D. Rodrigo, O. Limaj, D. Janner, D. Etezadi, F. J. García de Abajo, V. Pruneri, H. Altug, Science, 349, 165, 2015.

[2] R Stanley, Nature Photonics, 6, 409, 2012.

[3] F Neubrech, C Huck, K Weber, A Pucci and H Giessen, Chem. Rev., 117, 5110, 2017.

[4] C. Clavero, Nature Photonics, 8, 95, 2014.

[5] Tittl, A. et al., Adv. Mater. 27, 4597, 2015.

[6] Yan, H. et al., Nature Photon., 7, 394, 2013.

[7] G V. Naik, V M. Shalaev and A Boltasseva, Adv. Mater., 25, 3264, 2013.

[8] T M. Mattox, X Ye, K Manthiram, P. J Schuck, A. P Alivisatos and J J. Urban, Adv. Mater., 27, 5830, 2015.

[9] M. Wagner, A. S. McLeod, S. J. Maddox, Z. Fei, M. Liu, R. D. Averitt, M. M. Fogler, S. R. Bank, F. Keilmann, and D. N. Basov, Nano Lett., 14, 4529, 2014.

[10] J. B. Khurgin, Nature Nanotechnol., 10, 2, 2015.

[11] S. Law, V. Podolskiy and D. Wasserman, Nanophotonics, 2, 103, 2013.

[12] E Sachet, C T. Shelton, J S. Harris, B E. Gaddy, D L. Irving, S Curtarolo, B F. Donovan, P E. Hopkins, P A. Sharma, A L Sharma, J Ihlefeld, S Franzen and J-P Maria, Nature Mater., 14, 414, 2015.

[13] J. Wu, J. Appl. Phys., 106, 011101, 2009.

[14] P. D. C. King, T. D. Veal, P. H. Jefferson, S. A. Hatfield, L. F. J. Piper, C. F. McConville, F. Fuchs, J. Furthmüller, F. Bechstedt, Hai Lu and W. J. Schaff, Phys. Rev. B, 77, 045316, 2008. 
[15] A. Terentjevs, A. Catellani and G. Cicero, Appl. Phys. Lett., 96, 171901, 2010.

[16] G Fan, C Wang, J Fang, Nano Today, 9, 69, 2014.

[17] N S. Karan, Y Chen, Z Liu and R Beaulac, Chem. Mater. 28, 5601, 2016.

[18] J. C. Hsieh, D S Yun, E Hu and A M. Belcher, J. Mater. Chem., 20, 1435, 2010.

[19] J Choi and E. G. Gillan, J. Mater. Chem., 16, 3774, 2006.

[20] A Miura, T Takei and N Kumada, Cryst. Growth Des. 12, 4545, 2012.

[21] S. Zhao, S. Fathololoumi, K. H. Bevan, D. P. Liu, M. G. Kibria, Q. Li, G. T. Wang, H Guo and Z. Mi, Nano Lett., 12, 2877, 2012.

[22] C. Tessarek, S. Fladischer, C. Dieker, G. Sarau, B. Hoffmann, M. Bashouti, M. Göbelt, M. Heilmann, M. Latzel, E. Butzen, S. Figge, A. Gust, K. Höflich, T. Feichtner, M. Büchele, K. Schwarzburg, E. Spiecker and S. Christiansen, Nano Lett., 16, 3415, 2016.

[23] U. Kortshagen, Journal of Physics D: Applied Physics 42, 113001, 2009.

[24] A. Dixit, C. Sudakar, J. S. Thakur, K. Padmanabhan, S. Kumar, R. Naik, V. M. Naik, and G. Lawes, J. Appl. Phys., 105, 053104, 2009.

[25] V. M. Naik, R. Naik, D. B. Haddad, J. S. Thakur, G. W. Auner, H. Lu and W. J. Schaff, Appl. Phys. Lett. 86, 201913, 2005.

[26] P. K. B. Palomaki, E. M. Miller and N. R. Neale, J. Am. Chem. Soc. 135, 14142, 2013.

[27] J. M. Luther, P. K. Jain, T. Ewers and A. P. Alivisatos, Nature Mater. 10, 361, 2011.

[28] T. Klar, M. Perner, S. Grosse, G. von Plessen, W. Spirkl, J Feldmann, Phys. Rev. Lett. 80, 4249, 1998.

[29]B. L. Greenberg, S. Ganguly, J. T. Held, N. J. Kramer, K. A. Mkhoyan, E. S. Aydil and U. R. Kortshagen, Nano Lett., 15, 8162, 2015.

[30] S. D. Lounis, E. L. Runnerstrom, A. Bergerud, D. Nordlund, D. J. Milliron, J. Am. Chem. Soc., 136, 7110, 2014.

[31] E. L. Runnerstrom, A. Bergerud, A. Agrawal, R. W. Johns, C. J. Dahlman, A. Singh, S. M. Selbach and D. J. Milliron, Nano Lett., 16, 3390, 2016.

[32] X. Ye, J. Fei, B. T. Diroll, T. Paik and C. B. Murray, J. Am. Chem. Soc. 136, 11680, 2014.

[33] J. Wu, W. Walukiewicz, W. Shan, K. M. Yu, J. W. Ager, E. E. Haller, H Lu and W. J.

Schaff, Phys. Rev. B, 66, 201403, 2002.

[34] H. P. Maruska and J. J. Tietjen, Appl. Phys. Lett., 15, 327, 1969.

[35] M. G. Ganchenkova and R. M. Nieminen, Phys. Rev. Lett., 96, 196402, 2006

[36] L. F. J. Piper, T. D. Veal, C. F. McConville, H Lu, W. J. Schaf, Appl. Phys. Lett., 88, $252109,2006$. 
[37] I Pilch, D Söderström, MI Hasan, U Helmersson, N Brenning, App. Phys. Lett. 103, 193108, 2013.

[38] M I Hasan, I Pilch, D Soderstrom, D Lundin, U Helmersson and N Brenning, Plasma Sources Sci. Technol. 22, 035006, 2013.

[39] C. Rauch, F. Tuomisto, P. D. C. King, T. D. Veal, H. Lu, and W. J. Schaff, Appl. Phys. Lett. 101, 011903, 2012.

[40] D. C. Look, Appl. Phys. Lett. 80, 258, 2002.

[41] X. M. Duan and C. Stampfl, Phys Rev B, 77, 115207, 2008.

[42] R. E. Jones, S. X. Li, L. Hsu, K. M. Yu, W. Walukiewicz, Z. Liliental-Weber, J. W. Ager, E. E. Haller, H. Lu, W. J. Schaff, Physica B: Condensed Matter, 376-377, 436, 2006. 